\title{
OR7-002 - Pyrin 577 mutations in dominant autoinflammation
}

\author{
M Stoffels ${ }^{1,2,3^{*}}$, A Szperl ${ }^{4}$, A Simon ${ }^{1,2,3}$, MG Netea ${ }^{1,2,3}$, TS Plantinga ${ }^{1,2}$, M van Deuren ${ }^{1,2}$, S Kamphuis ${ }^{5}$, H Lachmann ${ }^{6}$, \\ E Cuppen ${ }^{7}$, WP Kloosterman ${ }^{7}$, J Frenkel ${ }^{8}$, CC van Diemen ${ }^{4}$, C Wijmenga ${ }^{4}, M_{\text {van Gijn }}^{7}$, JW van der Meer ${ }^{1,2,3}$ \\ From 7th Congress of International Society of Systemic Auto-Inflammatory Diseases (ISSAID) \\ Lausanne, Switerland. 22-26 May 2013
}

\section{Introduction}

Autoinflammatory disorders are disorders of the innate immune system. Standard genetic testing provided no correct diagnosis in a female patient from a nonconsanguineous family of British descent with a colchicineresponsive autosomal dominant periodic fever syndrome.

\section{Objectives}

We aimed to unravel the genetic cause of the symptoms in this family.

\section{Methods}

Whole exome sequencing was used to screen for novel sequence variants, which were validated by direct Sanger sequencing. Ex-vivo stimulations with peripheral blood mononuclear cells were done to study the functional consequences of the mutation. mRNA and cytokine levels were measured by q-PCR and ELISA, respectively.

\section{Results}

Whole exome sequencing revealed a novel missense sequence variant, not seen in around 6800 controls, mapping to exon 8 of the $M E F V$ gene (c. $1730 \mathrm{C}>\mathrm{A}$; p.T577N), co-segregating perfectly with disease in this family. Other mutations at the same amino acid (c.1730C>G; p.T577S; c.1729A >T; p.T577S) were found in a family of Turkish descent, with autosomal dominant inheritance of FMF-like phenotype, and a Dutch patient, respectively. Moreover, a mutation (c.1729A>G; p. T577A) was detected in 2 Dutch siblings, suffering from episodes of inflammation of varying severity not resembling FMF. PBMCs from one patient of the index family revealed increased basal IL-1 $\beta$ mRNA levels and

${ }^{1}$ Department of General Internal Medicine, Radboud University Nijmegen Medical Centre, Nijmegen, the Netherlands

Full list of author information is available at the end of the article cytokine responses after LPS stimulation. Responses normalized under colchicine treatment.

\section{Conclusion}

Heterozygous mutations at amino acid position 577 of pyrin can induce an autosomal dominant autoinflammatory syndrome. This suggests that T577, located in front of the C-terminal B30.2/SPRY domain, is crucial for pyrin function.

\section{Disclosure of interest}

M. Stoffels: None declared, A. Szperl: None declared, A. Simon Consultant for: Novartis and Swedish Orphan Biovitrum, M. Netea: None declared, T. Plantinga: None declared, M. van Deuren: None declared, S. Kamphuis: None declared, H. Lachmann: None declared, E. Cuppen: None declared, W. Kloosterman: None declared, J. Frenkel Consultant for: Novartis and Swedish Orphan Biovitrum, C. van Diemen: None declared, C. Wijmenga: None declared, M. van Gijn: None declared, J. van der Meer Consultant for: Novartis and Swedish Orphan Biovitrum.

\section{Authors' details}

${ }^{1}$ Department of General Internal Medicine, Radboud University Nijmegen Medical Centre, Nijmegen, the Netherlands. ${ }^{2}$ Nijmegen Centre for Infection, Inflammation and Immunity (N4i), Nijmegen, the Netherlands. ${ }^{3}$ Nijmegen Centre for Molecular Life Sciences, Nijmegen, the Netherlands. ${ }^{4}$ Department of Genetics, UMC Groningen, Groningen, the Netherlands. ${ }^{5}$ Sophia Children's Hospital, Erasmus UMC, Rotterdam, the Netherlands. 'Division of Medicine,

UK National Amyloidosis Centre, UCLMS, London, UK. Department of Medical Genetics, UMC Utrecht, Utrecht, the Netherlands. ${ }^{8}$ Division of Pediatrics, UMC Utrecht, Utrecht, the Netherlands.

\section{Published: 8 November 2013}

\section{doi:10.1186/1546-0096-11-S1-A103}

Cite this article as: Stoffels et al: OR7-002 - Pyrin 577 mutations in dominant autoinflammation. Pediatric Rheumatology 2013 11(Suppl 1): A103. 